\title{
SOLUTION OF MULTIPHASE HEAT CONDUCTION PROBLEMS VIA THE GENERALIZED INTEGRAL TRANSFORM TECHNIQUE WITH DOMAIN CHARACTERIZATION THROUGH THE INDICATOR FUNCTION
}

\begin{abstract}
H. A. Machado ${ }^{\mathrm{a}, \mathrm{b}, \mathrm{c}}$,
N. G. C. Leite ${ }^{\mathrm{a}}$,

E. Nogueira ${ }^{a}$,

and $\mathrm{H}$. Korzenowisk

${ }^{\mathrm{a}}$ Universidade do Estado do Rio de Janeiro Faculdade de Tecnologia

Rodovia Presidente Dutra, km 298

27537-000, Resende, RJ, Brasil nleite@uerj.br

bInstituto de Aeronáutica e Espaço

Praça Marechal Eduardo Gomes, 50

12228-904, São José dos Campos, SP, Brasil

'Universidade do Vale do Paraíba

Faculdade de Engenharia, Arquitetura e

Urbanismo, Av. Shishima Hifume, 2911

12244-000, São José dos Campos, SP, Brasil

Received: April 02, 2016

Revised: May 10, 2016

Accepted: June 12, 2016

ABSTRACT

The Generalized Integral Transform Technique (GITT) has appeared in the literature as an alternative to conventional discrete numerical methods for partial differential equations in heat transfer and fluid flow. This method permits the automatic control of the error and is easy to program, since there is no need for a discretization. The method has being constantly improved, but there still a vast number of practical problems that has not being solved satisfactory. In several brands of engineering, the transport equations have to be solved for a combination of different phases or materials or inside irregular domains. In this case, the mathematical resource of the Indicator Function can be employed. This function is a representation of the phases or parts of the domain with the numbers 0 and 1 for each phase. According to the method, the Indicator Function is defined by Poisson's equation, which is added to the system of the transport equations. An integral is done along the curve that defines the interface that will generate the source term in Poisson' equation used to calculate the Indicator Function distribution. The solution of the system of equations is done using the common GITT approach. Then, an analytical expression for each transformed potential of the indicator function and the other variables are available. Once the transformed potentials are known, the Indicator Function can be analytically operated, and the interface can be represented by an analytical continuous function. In this work, the use of the GITT in conjunction with the Indicator Function is proposed. The methodology is described and some previous results are presented. GITT is applied to a two-dimensional heat conduction problem in a multiphase domain with an irregular geometry, inside a square domain. The methodology presented here can be extended to all brands of convection-diffusion problems already solved via GITT.
\end{abstract}

Keywords: integral transforms, irregular geometries, heat conduction

\section{NOMENCLATURE}

I indicator function

n normal unitary vector

p domain perimeter

Q dimensionless source term

$\mathrm{T}$ dimensionless temperature

$\mathrm{t}$ dimensionless time

$\mathrm{x}, \mathrm{y}$ dimensionless Cartesian coordinates

\section{Greek symbols}

$\alpha \quad$ dimensionless thermal diffusivity

$\theta \quad$ transformed potential of temperature

$\Phi \quad$ Indicator Function eigenfunction

$\Gamma \quad$ temperature eigenfunction

$\phi \quad$ indicator function eigenvalue

$\lambda$ temperature eigenvalue

\section{Subscripts}

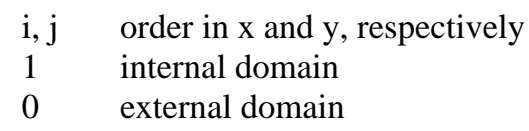

\section{INTRODUCTION}

The Generalized Integral Transform Technique (GITT) has appeared in the literature (Cotta, 1993) as an alternative to conventional discrete numerical methods for partial differential equations in heat and fluid flow. Its hybrid numerical-analytical structure permits the automatic control of the global error in the simulation, which avoids the need for several computer program runs to inspect for the convergence on the final results, yielding codes that automatically work towards user prescribed accuracy targets. This method is also easy to program, since there is no need for a discretization mesh and its adaptive refinement according to the potential field and physical situation to be calculated. 
The method has being constantly improved in order to solve convection-diffusion problems with increasing complexity, like the solution of the Navier-Stokes equations in internal flows inside irregular geometries (Monteiro et al, 2004, Silva et al, 2004), new eigenvalue problems with optimization of the transformed potentials (Guigon et al, 2004) and other successfully examples. However, there still a vast number of practical problems that has not been solved satisfactory by the method, considering the strong concurrence of the traditional discrete methods. One of the main difficult in GITT use for engineering problems is the need for previous algebraic treatment of the equations, which restricts its popularization, in spite of the advantages presented concerned to the error control and no mesh generation. As a consequence, some classes of problems have not been studied with this method yet, like two-dimensional phase change, complex twophase flow and irregular domains, etc.

In several brands of engineering, the transport equations have to be solved for a combination of different phases or materials, or considering the presence of discrete source terms. In this case, the simple application of the well-known discrete numerical methods demands some specific treatment: the discontinuity must be isolated via coordinate or domain transformation or grid refinement. Both are complex numerical processes, and add some residual error to final results. In this case, the mathematical resource of the Indicator Function, as defined in the Interface Tracking Method of Unverdi and Tryggvason (1992) and Juric and Tryggvason (1998) can be employed. This function is a representation of the phases or parts of the domain with the numbers 0 and 1 for each phase (in this point it is supposed exists only two phases, however this method can be used in presence of n-phases).

This work is an extension of the previous work of Machado and Leite (2013) where this methodology was applied in a one-phase domain. In this work GITT is applied to a two-dimensional heat conduction problem in a square domain with two phases separated by an irregular edge. A constant source term is applied in one phase and some previous results are presented, where the convergence characteristics and a comparison with the exact solution are described.

\section{FORMULATION}

Consider a two phase square domain with unitary side where a pure conduction heat transfer occurs. The energy equation with all variables in dimensionless form will be:

$$
\frac{\partial \mathrm{T}}{\partial \mathrm{t}}=\nabla \cdot[\alpha(\mathrm{x}, \mathrm{y}) \cdot \nabla \mathrm{T}]+\mathrm{Q}(\mathrm{x}, \mathrm{y}, \mathrm{t})
$$

Where $Q$ is a source term and $\alpha(x, y)$ is the thermal diffusivity of both regions corresponding to each phase. This property is calculated using the Indicator Function $-I(x, y)$, and is given as $\alpha(x, y)=$ $\alpha_{0}+\left(\alpha_{1}-\alpha_{0}\right) \mathrm{I}(\mathrm{x}, \mathrm{y}) ; \alpha_{0}$ is the thermal diffusivity for the region where $I=0$ and $\alpha_{1}$ for the region where $I$ $=1$.

Consider also prescribed wall temperatures and initial condition equal to zero. In order to apply GITT, an associated eigenvalue problem is chosen, that has similar boundary conditions. This problem will be the Sturm-Liouville problem:

$$
\Gamma_{\mathrm{i}}^{\prime \prime}=-\lambda_{\mathrm{i}}^{2} \Gamma_{\mathrm{i}} \text { for } 0 \leq \mathrm{x} \leq 1 \text { or } 0 \leq \mathrm{y} \leq 1
$$

with an analytical solution known for the eigenfunctions $\Gamma_{i}(x)$, eigenvalues $\lambda_{i}$ and norms $N_{i}$. The boundary condition used is prescribed wall temperature, below.

$$
\Gamma_{\mathrm{i}}(0)=0 ; \Gamma_{\mathrm{i}}(1)=0
$$

The solution allows knowing the spatial dependency of the solution analytically. Since for this case the boundary conditions are the same in the whole border, the same eingenfunctions are applied in both directions, $x$ and $y$.

Next step is to define the Transformed-Inverse pair:

$$
\begin{gathered}
\Theta_{\mathrm{ij}}(\mathrm{t})=\int_{0}^{1} \int_{0}^{1} \bar{\Gamma}_{\mathrm{i}}(\mathrm{x}) \bar{\Gamma}_{\mathrm{j}}(\mathrm{y}) \mathrm{T}(\mathrm{x}, \mathrm{y}, \mathrm{t}) \mathrm{dyd} \mathrm{x} \\
\mathrm{T}(\mathrm{x}, \mathrm{y}, \mathrm{t})=\sum_{\mathrm{i}=1}^{\infty} \sum_{\mathrm{j}=1}^{\infty} \bar{\Gamma}_{\mathrm{i}}(\mathrm{x}) \bar{\Gamma}_{\mathrm{j}}(\mathrm{y}) \Theta_{\mathrm{ij}}(\mathrm{t})
\end{gathered}
$$

where $\bar{\Gamma}_{i}$ is the normalized eigenfunction:

$$
\bar{\Gamma}_{\mathrm{i}}=\Gamma_{\mathrm{i}} / \mathrm{N}_{\mathrm{i}}^{1 / 2}
$$

Applying the integral operator $\int_{0}^{1} \int_{0}^{1} \bar{\Gamma}_{\mathrm{i}}(\mathrm{x}) \bar{\Gamma}_{\mathrm{j}}(\mathrm{y})_{-}$dydx over Eq. (1), substituting $T$ by its definition in the Eq. (3.b) and taking advantage of the ortogonality of the eigenfunction, according to Mikhailov and Özisik (1984), one obtain:

$$
\begin{aligned}
& \frac{\mathrm{d} \Theta_{\mathrm{ij}}(\mathrm{t})}{\mathrm{dt}}=-\alpha_{0}\left(\lambda_{\mathrm{i}}^{2}+\lambda_{\mathrm{j}}^{2}\right) \Theta_{\mathrm{ij}}(\mathrm{t})+ \\
& \int_{0}^{1} \int_{0}^{1} \bar{\Gamma}_{\mathrm{i}}(\mathrm{x}) \bar{\Gamma}_{\mathrm{j}}(\mathrm{y})\left[\left(\alpha_{1}-\alpha_{0}\right) \cdot \mathrm{I}(\mathrm{x}, \mathrm{y}) \cdot \nabla^{2} \mathrm{~T}+\mathrm{Q}(\mathrm{x}, \mathrm{y}, \mathrm{t})\right] \mathrm{dydx}
\end{aligned}
$$

The resulting infinite system of coupled ordinary differential equations will be truncated to an order NT high enough to assure the precision target, and represented as a first order matrix system 
$d \boldsymbol{Y} / d t=\boldsymbol{D}(\boldsymbol{Y}, t)$, where the vector $\boldsymbol{Y}$ represents the transformed potentials $\Theta i j(i, j=1,2, \ldots, N T)$. According to the shape of the source term $Q$ or the variation of thermal conductivity $\alpha$, the system allows straight analytical solutions. This is not the case, since the spatial dependence of both terms is arbitrary, and the system is coupled. The infinite summation used to represent the original potential, Eq. (3.b), will be truncated to the same NT order. Initial conditions for transformed potentials are obtained from the transformation of the original ones.

This system is stiff, where the frequency of each solution is quite different, and has to be solved by specific computational libraries, like DIVPAG, from IMSL library (1989), based on the Gear's method. Once the transformed potentials are obtained for each time in a marching process, the original potential can be recovered through Eq. (3.b), since spatial dependence is analytically known.

The Indicator Function is defined by Poisson's equation:

$$
\nabla^{2} \mathbf{I}=\nabla \mathbf{G}
$$

Vector $\boldsymbol{G}$ is the distribution of interface over the domain, and is given as:

$$
\mathbf{G}=\int_{\mathrm{S}} \mathbf{n} \delta\left(\mathbf{x}-\mathbf{x}_{\mathbf{i}}\right) \mathrm{ds}
$$

where $\boldsymbol{n}$ is the normal vector along the interface, $\delta$ is the Dirac function, which is nonzero only when $\boldsymbol{x}=$ $\boldsymbol{x}_{\boldsymbol{f}}$ (subscript $f$ denotes points along interface). The integral over $s$ is done along the curve that defines the interface.

The solution of Eq. (6) is done using the common GITT approach. Function $I(x, y)$ is expanded in the Transformed-Inverse pair, as temperature in Eq. (3). The eigenfunctions $\Phi_{i}(x$ or $y)$ for $I(x, y)$ are obtained from the same auxiliary problem as temperature, Eq. (2.a,c), using zero flux as boundary condition in both directions. Next step is apply the integral operator $\int_{0}^{1} \int_{0}^{1} \bar{\Phi}_{i}(x) \bar{\Phi}_{j}(y) \_d y d x$ and again replacing $\mathrm{I}(\mathrm{x}, \mathrm{y})$ by its expansion, yielding:

$$
-\left(\phi_{i}^{2}+\phi_{j}^{2}\right) \cdot \bar{I}_{i j}=\int_{0}^{1} \int_{0}^{1} \bar{\Phi}_{i}(x) \bar{\Phi}_{j}(y) \nabla \cdot G \cdot d x d y
$$

where $\phi_{i}$ or $j$ are the eigenvalues for each direction. From Eq. (6) and after some manipulation, including the use of the chain rule, we finally obtain an analytically expression for the transformed potential of the Indicator Function:

$$
\begin{aligned}
& -\left(\phi_{\mathrm{i}}^{2}+\phi_{\mathrm{j}}^{2}\right) \cdot \overline{\mathrm{I}}_{\mathrm{ij}}= \\
& -\int_{0}^{\mathrm{p}}\left[\mathrm{n}_{\mathrm{x}}(\mathrm{p}) \cdot \Phi_{\mathrm{i}}^{\prime}(\mathrm{x}) \cdot \Phi_{\mathrm{j}}(\mathrm{y})\right. \\
& \left.+\mathrm{n}_{\mathrm{y}}(\mathrm{p}) \cdot \Phi_{\mathrm{j}}^{\prime}(\mathrm{y}) \cdot \Phi_{\mathrm{i}}(\mathrm{x})\right] \mathrm{d} \mathrm{p}
\end{aligned}
$$

Equation (9) provides an analytical expression for each transformed potential of the indicator function. The integral at the right hand side is done over the parameter $p$, that commonly can be considered the length along the curve which defines the interface, and $n_{x}, n_{y}$ are the components of the normal vector. The length is calculated defining a beginning for the curve. One should observe that the curve must be closed, and the two regions have to be completely separated.

Once the transformed potentials are known, the Indicator Function can be analytically operated, and the interface can be represented by an analytical continuous function, in a similar form as a Fourier series. The interface will have a thin thickness, which will be smaller as a higher number of terms are used in the expansion. Within this thickness, $I(x, y)$ will present values varying from 0 to 1 .

\section{MATHEMATICAL MODEL}

The domain of calculation where the determination of the temperature field will be performed is showed in Fig.1. The square inside the domain corresponds to one phase where the Indicator Function is equal to 1 and in the rest of the physical domain (outside the small square) the Indicator function will be equal to zero. A prescribed heat source term is applied in the phase corresponding to $I$ $=1$ while the other is kept at constant temperatures equal to zero in the border. Values assumed for $\alpha$ were 1 inside the internal square and 0 outside it. The square side was $L=0.5$ and the imposed heat source was $Q=100$. Initial temperatures for both phases were taken as zero.

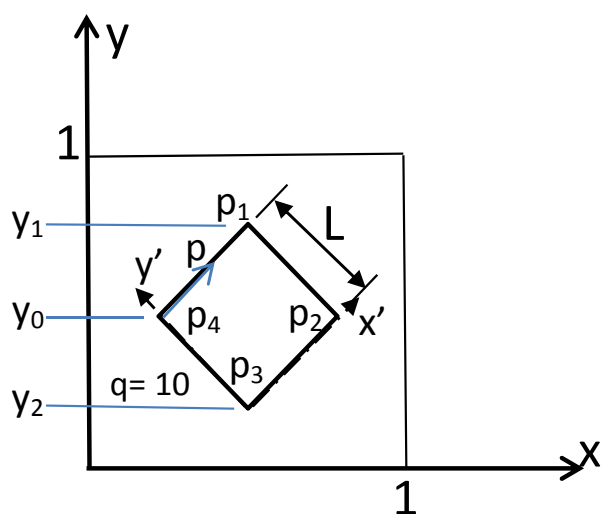

Figure 1. Schematics of the physical domain used.

This problem admits an exact solution extracted from the energy conservation law:

$\mathrm{T}(\mathrm{x}, \mathrm{y}, \mathrm{t})=\mathrm{Qt}$ for the phase where $\mathrm{I}=1$

$T(x, y, t)=0$ for the phase where $I=0$

Since temperature and Indicator Function have 
the same boundary condition (prescribed $\mathrm{T}$ and $\mathrm{I}$ equal to zero at the borders) they have the same eigenvalue problem. Consequently the transformation of the source term in Eq.(5) yields:

$$
\begin{aligned}
& \frac{\mathrm{d} \Theta_{\mathrm{ij}}(\mathrm{t})}{\mathrm{dt}}=-\alpha_{0}\left(\lambda_{\mathrm{i}}^{2}+\lambda_{\mathrm{j}}^{2}\right) \Theta_{\mathrm{i}, \mathrm{j}}(\mathrm{t})+ \\
& \int_{0}^{1} \int_{0}^{1} \bar{\Gamma}_{\mathrm{i}}(\mathrm{x}) \bar{\Gamma}_{\mathrm{j}}(\mathrm{y})\left(\alpha_{1}-\alpha_{0}\right) \cdot \mathrm{I}(\mathrm{x}, \mathrm{y}) \cdot \nabla^{2} \mathrm{Tdydx} \\
& +\overline{\mathrm{I}}_{\mathrm{i}, \mathrm{j}} \mathrm{Q}
\end{aligned}
$$

In order to perform the integral in Eq.(9), the interface between the internal square and the external domain is described through the combination of two functions:

$$
\begin{gathered}
y= \begin{cases}\mathrm{f}_{1} & \text { for } \mathrm{y}_{0}<\mathrm{y}<\mathrm{y}_{1} \\
\mathrm{f}_{2} & \text { for } \mathrm{y}_{0}<\mathrm{y}<\mathrm{y}_{2}\end{cases} \\
\mathrm{f}_{1}= \begin{cases}\mathrm{x}(\mathrm{p})+\mathrm{b}_{1} & \text { for } 0<\mathrm{p}<\mathrm{p}_{1} \\
-\mathrm{x}(\mathrm{p})+\mathrm{b}_{2} & \text { for } \mathrm{p}_{1}<\mathrm{p}<\mathrm{p}_{2}\end{cases} \\
\mathrm{f}_{2}= \begin{cases}-\mathrm{x}(\mathrm{p})+\mathrm{b}_{3} & \text { for } \mathrm{p}_{2}<\mathrm{p}<\mathrm{p}_{3} \\
\mathrm{x}(\mathrm{p})+\mathrm{b}_{4} & \text { for } \mathrm{p}_{3}<\mathrm{p}<\mathrm{p}_{4}\end{cases}
\end{gathered}
$$

\section{RESULTS AND DISCUSSION}

Applying the integral transform to the Poisson's Equation, the distribution of the indicator function is obtained for the whole domains of calculation. In order to analyze the convergence of the solution, different values for the number of terms of the expansion were used. Figures 2-4 shows the distribution of $I(x, y)$ for 3 values of $n$ (number of terms used).

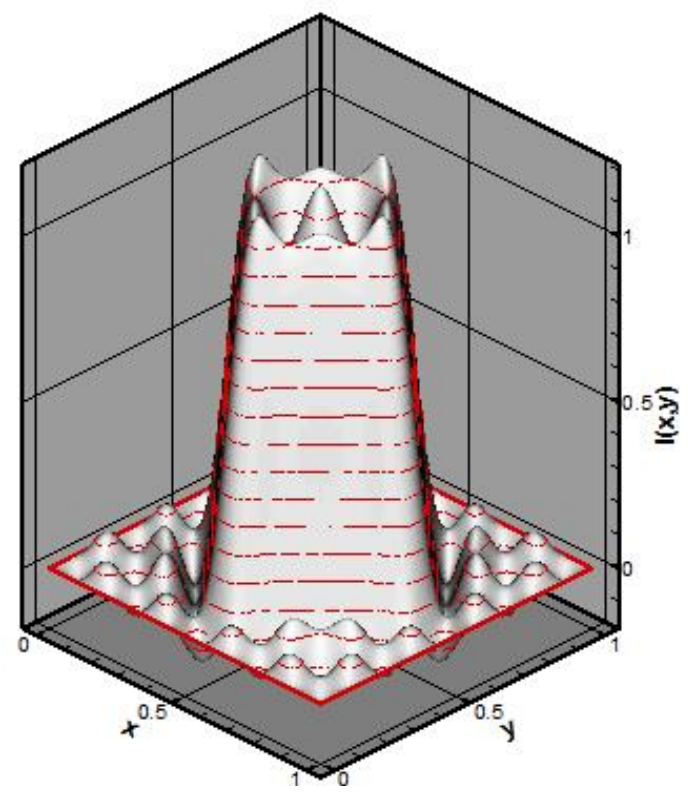

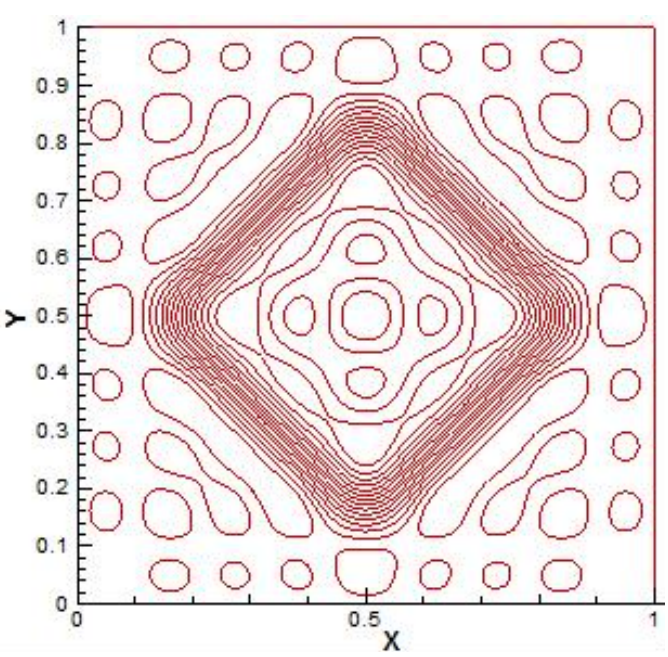

Figure 2. Distribution of Indicator Function in the domain of calculation, 3-D and 2-D views, for $\mathrm{n}=10$.
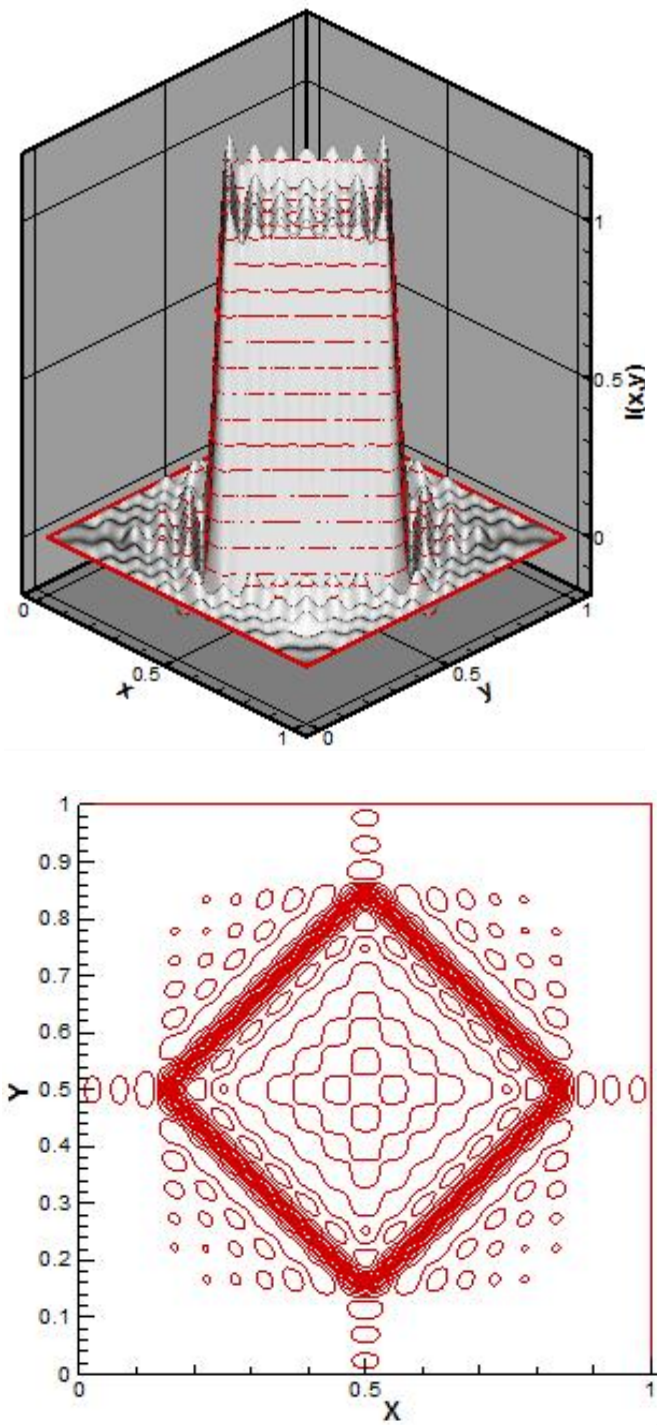

Figure 3. Distribution of Indicator Function in the domain of calculation, 3-D and 2-D views, for $n=20$. 

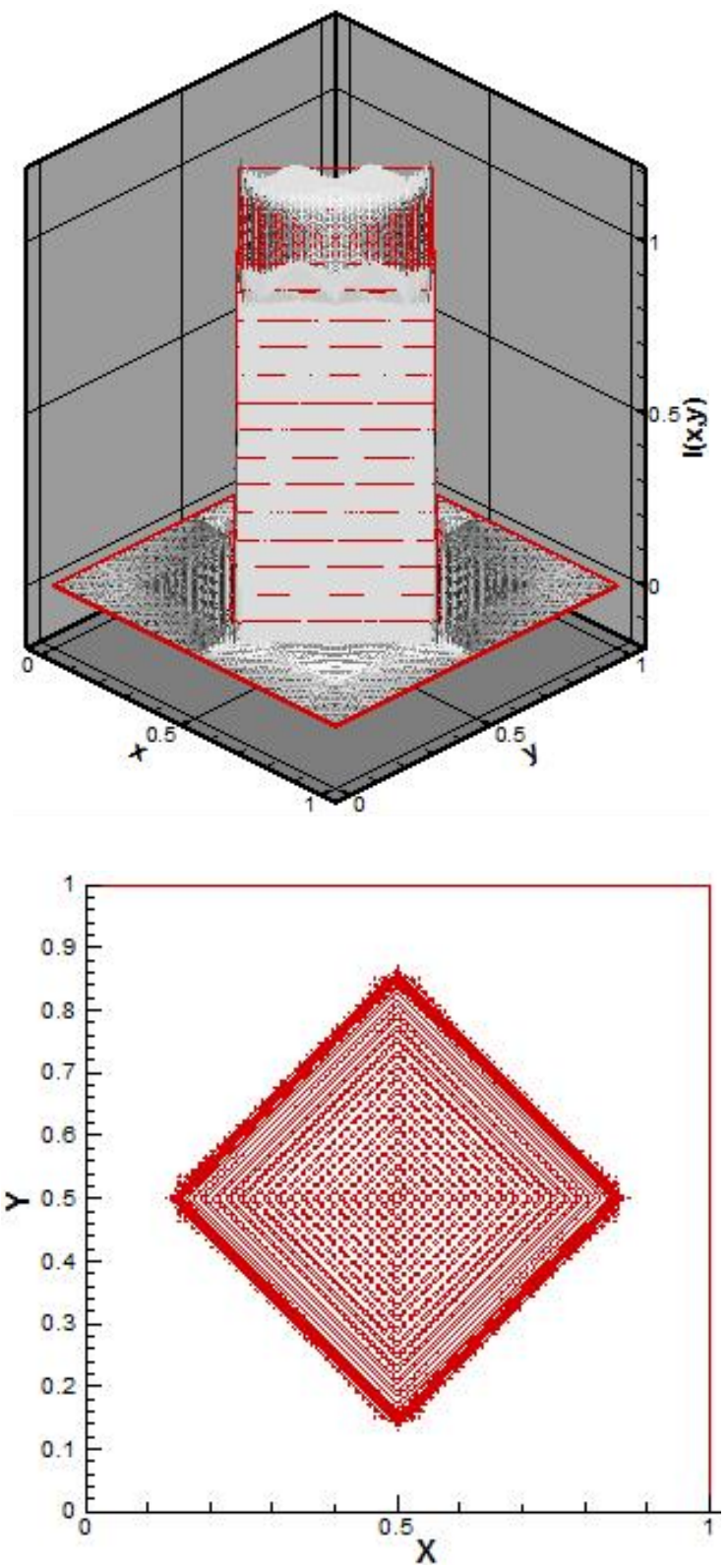

Figure 4. Distribution of Indicator Function in the domain of calculation, 3-D and 2-D views, for $n=$ 100.

The sequence shows the thickness reduction of the interface region with the rising in the number of terms of the expansion. Figure 5 shows the direct comparison, extracted toward the diagonal of the internal square $(y=0.5)$. In this case, behind the reduction in the interface thickness (what corresponds in a reduction in the slope of the interface), the oscillation amplitude also reduces. However, even with 100 terms, the peaks in the interface are still high, compared to the rest of the domains. It seems that the derivatives in the interface region do not present a good precision.
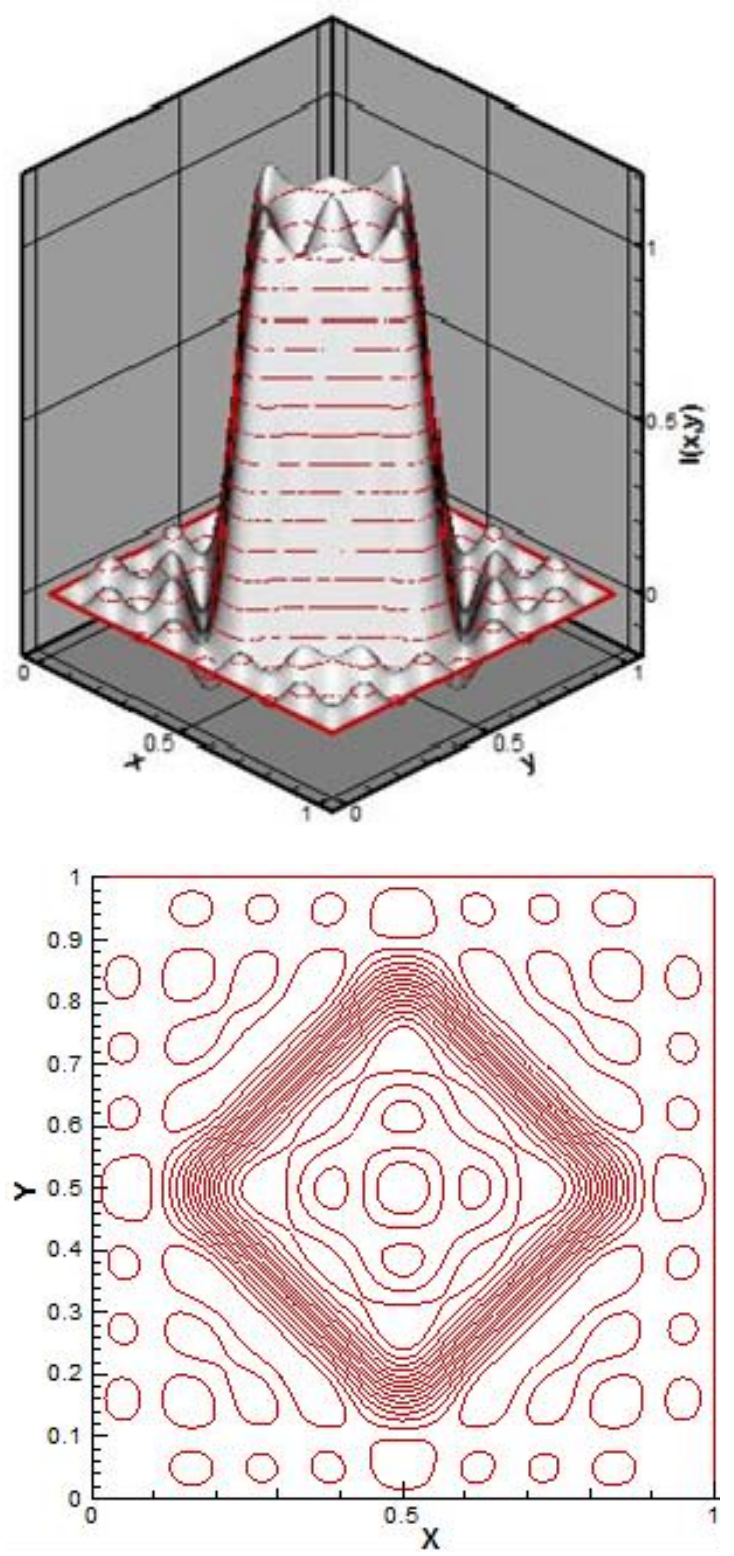

Figure 2. Distribution of Indicator Function in the domain of calculation, 3-D and 2-D views, for $\mathrm{n}=10$.

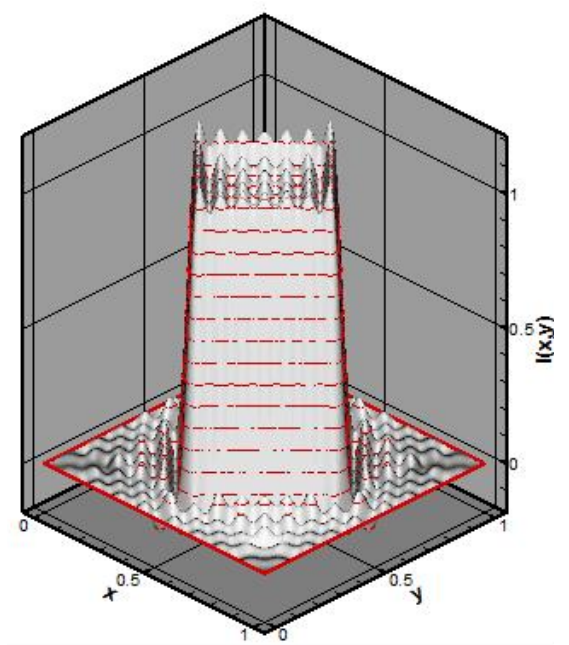




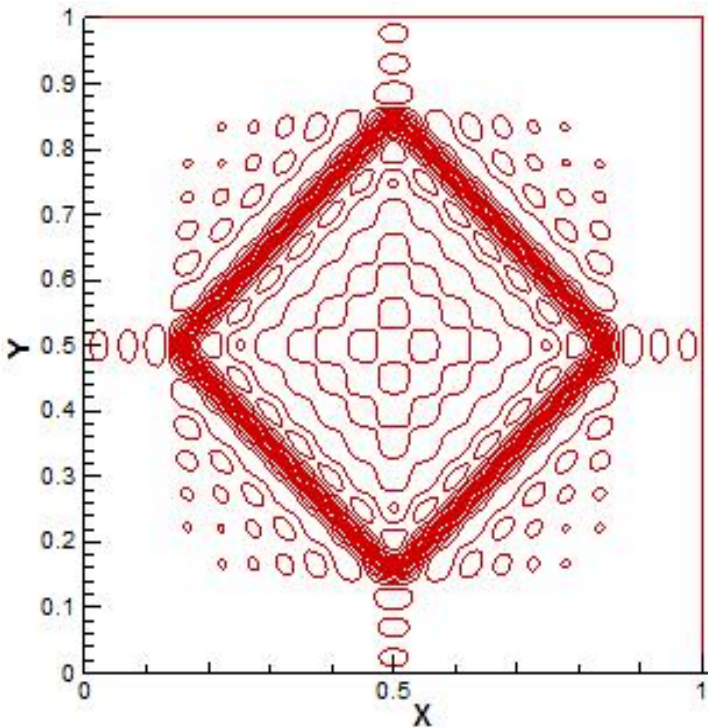

Figure 3. Distribution of Indicator Function in the domain of calculation, 3-D and 2-D views, for $n=20$.

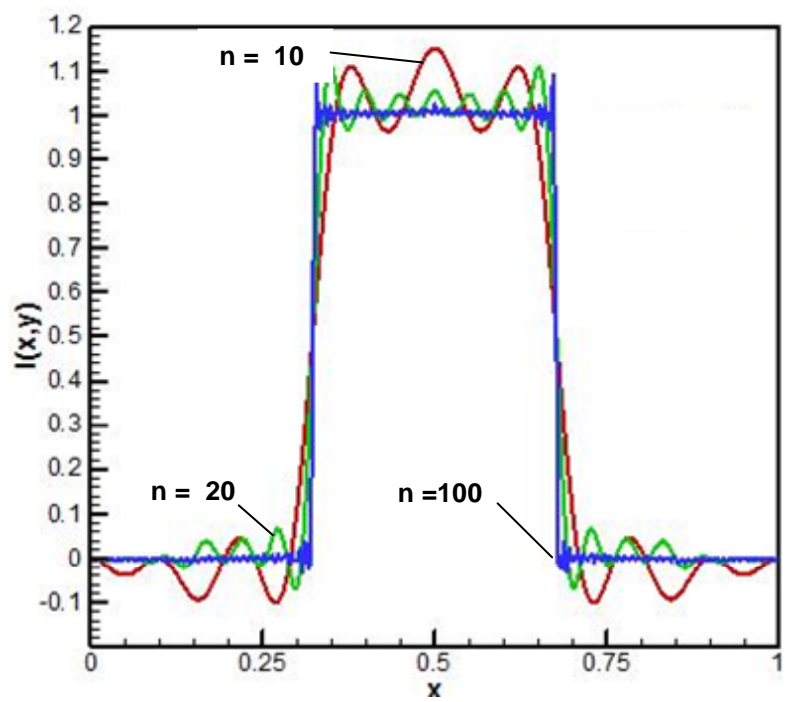

Figure 5. Convergence of Indicator Function, for $\mathrm{y}=$ 0.5 .

The method of solution was applied to the heat conduction problem, for $n=10,20,30$ and 40 . Results for temperature distribution are showed in Figs.6. In Figure 7, the convergence of the GITT solution is showed in the $x$ coordinates at $y=0.5$ and $\mathrm{t}=0.01$. The solution is compared with the exact solution. Although the results for GITT are still far from the right solution, it seems to be toward this result, and presents the same behavior. In Figure 6, the lack of convergence and the effect of the oscillation are clear in the phases interface. The discontinuity at that point is better represented as more terms are added to the solution, reducing the interface slope.

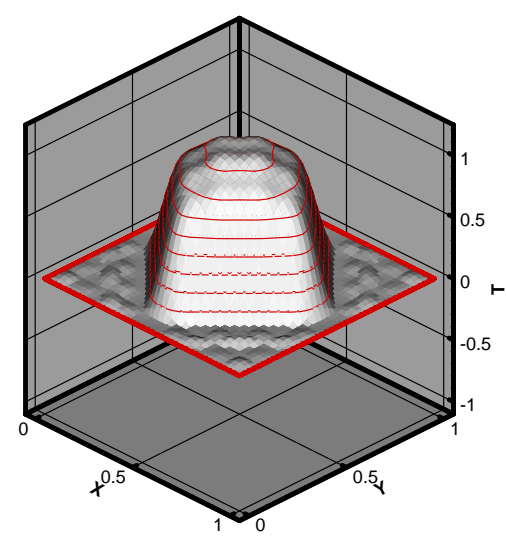

a) $n=10$

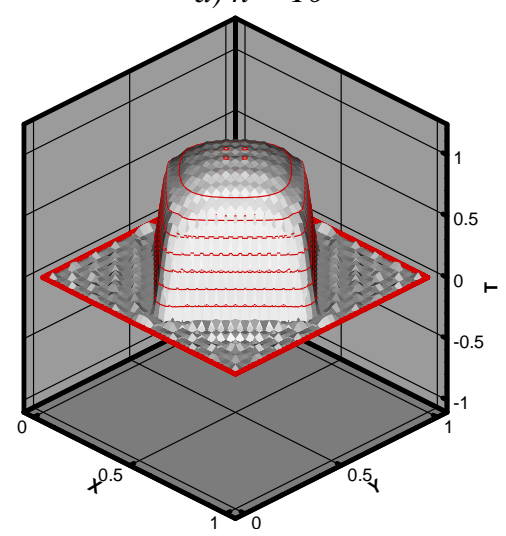

b) $n=20$

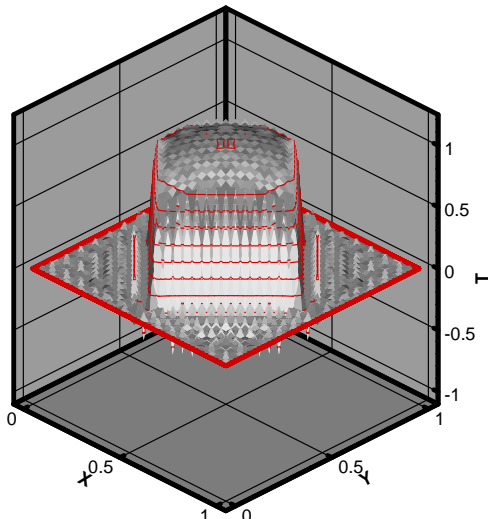

c) $n=30$

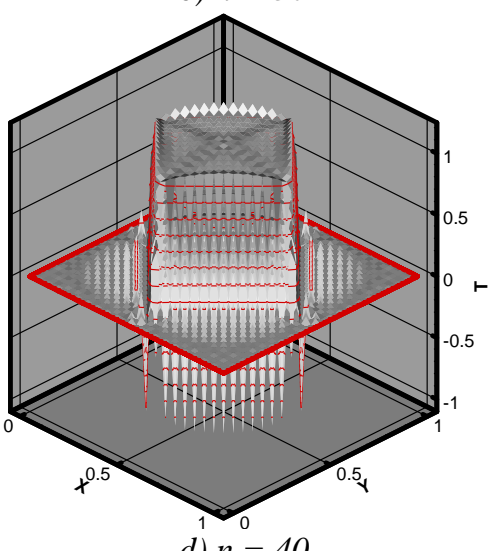

d) $n=40$

Figure 6. Temperature distribution for $\mathrm{t}=0.01$. 


\section{CONCLUSIONS}

In this work, the GITT was employed to solve a pure heat conduction problem with prescribed source term in an irregular domain with two phases, represented through the use of the indicator function. The domain was well represented by the indicator function field, obtained through the integral transform. However, the solution for the temperature distribution was still far from a good result, when compared with the exact solution. Although a good agreement was not obtained for the GITT, the qualitative behavior was considered in agreement with the right solution. An improvement in the convergence and the reduction with the oscillation would result in a very reliable method for heat conduction problems in irregular domains, considering the advantages in its implementation.

\section{REFERENCES}

Cotta, R. M., 1998, The Integral Transform method in thermal and Fluids Science and Engineering, Begell House.

Gonçalves, C. V., Arruda, J. M., Lebron, S. C. and Machado, H. A., 2000.a, Simulation of Punctual Heat Sources via Integral Transforms, in: Proceedings of the IV SIMMEC, Uberlândia.

Gonçalves, C. V., Arruda, J. M., Lebron, S. C., Ribeiro, C. R., and Machado, H. A., 2000.b, Comparative Study using Discrete and SemiAnalytical Methods in the Simulation of Punctual Heat Sources, in: Proceedings of CILANCE, Rio de Janeiro.

Guigon, J. M. B., Pérez-Guerrero, J. S., and Cotta, R. M., 2004, Computation Optimization of Integral Transform Algorithms for the Lid-Driven Cavity Flow Problem, in: Proceedings of the ENCIT 2004, Rio de Janeiro.

Juric, D., and Tryggvason, G., 1998, Computations of Boiling flows, International Journal of Multiphase Flow, Vol. 24, No. 3, pp. 387-410.

Machado, H. A., 2001, An Efficient Numerical Integration Algorithm for Integral Transform Solution of Nonlinear Convection-Difusion Problems, Hybrid Methods in Engineering, New York, Vol. 2, No. 4, pp. 451-461.

Machado, H. A., and Dantas, L. B., 2001, Solution of Heat Conduction Problems with Discontinuities via Integral Transforms, in: Proceedings of the 2nd ICCHMT, Rio de Janeiro.

Machado, H. A., and Leite, N. G. C., 2013, Solution of Multiphase Heat Conduction Problems Via the Generalized Integral Transform Technique with Domain Characterization Through the Indicator Function, in: Proceedings of the COBEM 2013, Ribeirão Preto.

Monteiro, E. R., Macedo, E. N., Quaresma, J. N. N., and Cotta, R. M., 2004, A Solution via Integral Transforms for Fully Developed Flow in Doubly
Connected Ducts, in: Proceedings of the ENCIT 2004, Rio de Janeiro.

Pontedeiro, A., Cotta, R. M., and Jian, S., 2008, Improved Lumped Model for Thermal Analysis of High Burn-up Nuclear Fuel Rods, Progress in Nuclear Energy, Vol. 50, pp. 767-773

Silva, R. L., Quaresma, J. N. N., and Dos Santos, C. A. C., 2004, Hybrid Solution for Flow Development in Irregular Ducts, in: Proceedings of the ENCIT 2004, Rio de Janeiro.

Unverdi, S. O., and Tryggvason, G., 1992, A Front-Tracking Method for Viscous, Incompressible, Multi-fluid Flows, Journal of Computational Physics, Vol. 100, pp. 25-37. 